\title{
PYODERMA GANGRENOSUM AT CAESAREAN INCISION SITE RESPONSE TO DEXAMETHASONE THERAPY
}

Sindhuri K르. Kallappa Herekal², Kariji Siddalingappa ${ }^{3}$, Jasveen Kaur ${ }^{4}$

\section{HOW TO CITE THIS ARTICLE:}

Sindhuri K, Kallappa Herekal, Kariji Siddalingappa, Jasveen Kaur. "Pyoderma Gangrenosum at Caesarean Incision Site Response to Dexamethasone Therapy". Journal of Evolution of Medical and Dental Sciences 2014; Vol. 3, Issue 65, November 27; Page: 14214-14216, DOI: 10.14260/jemds/2014/3903

INTRODUCTION: Pyoderma gangrenosum (PG) is a primarily sterile inflammatory neutrophilic dermatosis; characterized by recurrent cutaneous ulcerations with mucopurulent or hemorrhagic exudate. ${ }^{1}$ In many cases, PG is associated with systemic diseases like ulcerative colitis, Crohn's disease, polyarthritis, monoclonal gammopathy or other conditions. ${ }^{2}$

KEYWORDS: Pyoderma gangrenosum, dexamethasone.

CASE REPORT: A 27 year old woman presented on $7^{\text {th }}$ post-operative day of caesarian section with extremely painful ulcer at the incision site. There was rapid increase in its size. She had history of typhoid fever 15 days back and no similar complaints in the past.

Clinical examination showed a solitary ulcer measuring about $10 \mathrm{X} 5 \mathrm{~cm}$ with undermined edge, violaceous border, and necrotic base with fulminant peripheral extension (Figure 1). On palpation, extreme tenderness and indurated edge was noted. General physical examination showed no abnormality. Investigations like complete blood count, renal and liver profile were within normal limits. Grams staining and culture from the ulcer showed no organisms. Histopathology was not done as patient can't afford Auto-antibodies were negative. Pathergy test was positive. Colonoscopy could not be done in our patient. Ulcerative HSV infection, vascular occlusive or venous disease, vasculitis, and bacterial infection were considered as differential diagnosis. In view of clinical and laboratory investigations, we labeled the condition as pyoderma gangrenosum.

Treatment was instituted with systemic dexamethasone for two weeks, and oral steroids were given in tapering doses for next 6 weeks. Wound care was done with gentle cleansing, hydrocolloid dressings. Antibacterial agent like silver sulfadiazine was used once daily and tacrolimus with placental extract gel was applied at night. Patient showed marked improvement of ulcer after 3 weeks of treatment (Figure 2). Ulcer healed with scar formation at the end of 8 weeks (Figure 3).

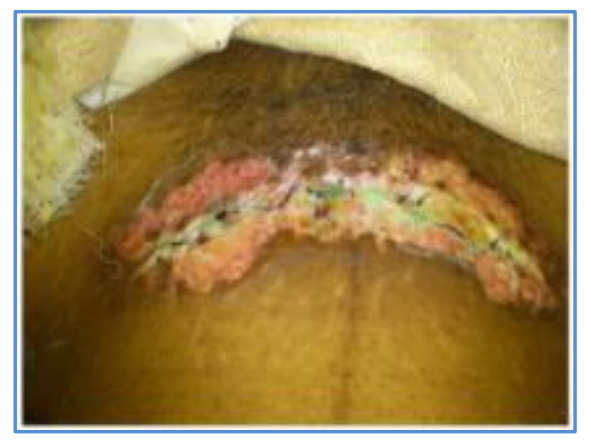

Fig. 1: At the time of admission after $7^{\text {th }}$ day of cesearion

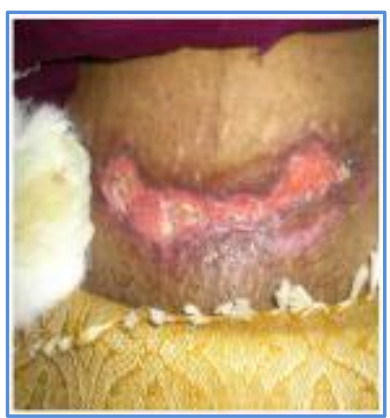

Fig. 2: 3 weeks after admission 


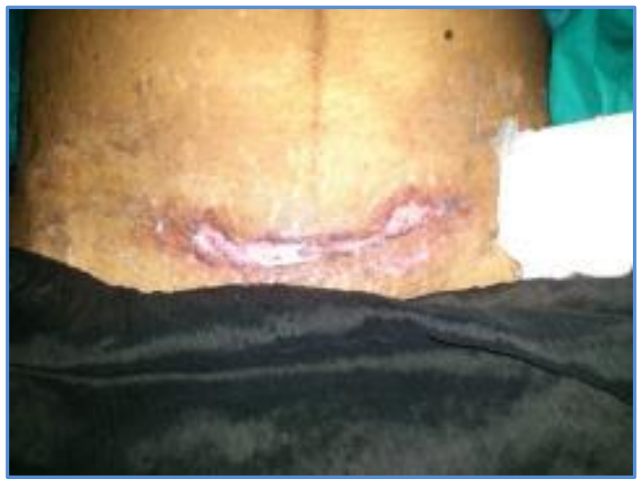

Fig. 3: 1 month after admission

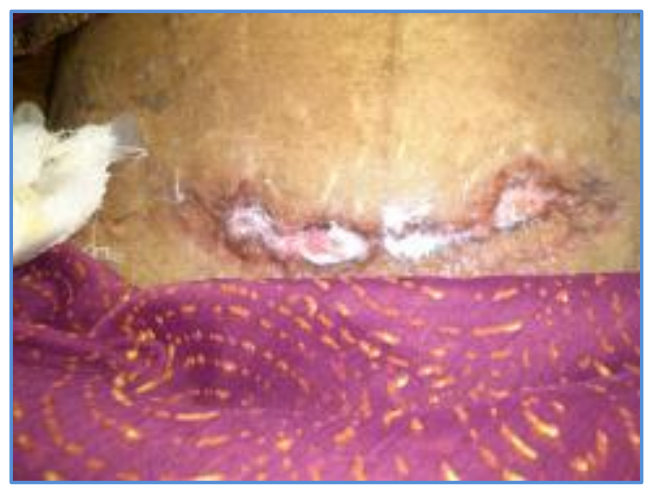

Fig. 4: At the time of discharge

DISCUSSION: Pyoderma gangrenosum first described by Brunsting et $\mathrm{al}^{3}$ in 1930, is a destructive, inflammatory neutrophilic dermatosis. Several clinical variants have been described in PG and are easy to misdiagnose. Variants of PG include classic PG, peristomal, pustular, bullous, vegetative and ulcerative types. ${ }^{1}$

Pyoderma gangrenosum lesions have a predilection for the pretibial areas, although they can affect any other body site including oral, genital mucosa, and viscera such as lung or spleen. A typical lesion would be a follicular pustule that ulcerates and is surrounded by erythematous, edematous skin. The ragged, undermined, and violaceous or bluish ulcer border are distinctive of this condition and key in making the diagnosis. ${ }^{4}$

Diagnosis of PG is based on a history of an underlying disease, typical clinical presentation, histopathology, and exclusion of other ulcerative diseases. The histopathology of PG is nonspecific and changes with the stage of lesion. The initial lesions show a deep suppurative folliculitis with dense neutrophilic infiltrate. In about $40 \%$ of cases, leukocytoclastic vasculitis is present ${ }^{5}$. At the edge of the ulcer, there is a perivascular lymphocytic infiltrate and fibrinoid necrosis of the dermal vessel wall with occasional extravasation of RBCs and thrombosis is also seen. ${ }^{6}$

Differential diagnosis varies with different clinical variant of PG. In the early stages, nonulcerative lesions including nodules, pustules and bullous formations should be distinguished from skin conditions like folliculitis, necrotizing vasculitis, erythema multiforme, abscesses and tick bites. For ulcerative lesions of $P G$, frequent differential diagnosis include infective such as necrotizing fascitis, vascular occlusive or venous disease, necrobiosis lipoidica, livedoid vasculitis, sarcoidosis, or cutaneous malignancies such as squamous cell carcinoma and basal cell carcinoma, vasculitic processes such as Wegener's, malignancy such as lymphoma or leukemia, and pustular drug reactions. $^{7}$

Various treatment strategies have been described for the management of PG. The aim of therapy is to halt the progression of the ulcers, promote healing, reduce pain, and minimize the resulting scarring. Immunosuppression is the mainstay of treatment and high dose corticosteroids is the first line of treatment. In steroid refractory cases, other immunosuppressive drugs found to be effective are Cyclosporine A, Azathioprine, Cyclophosphamide, Chlorambucil, and Mycophenolate mofetil. ${ }^{6}$ Drugs with anti-inflammatory actions are also found to be effective in few individuals; commonly used drugs in this category are dapsone, clofazimine, minocycline, and thalidomide. ${ }^{4}$ 
More recently, pyoderma gangrenosum was reported to resolve after treatment with biologics as infliximab, etanercept, and oral as well as topical tacrolimus, therapeutic plasma exchange, intravenous immunoglobulins, and hyperbaric oxygen. ${ }^{4}$ In addition to systemic therapy, local wound care with cleansing, use of antiseptic agents and certain specific agents like topical tacrolimus, hyauronic acid, becaplermin, lyophilized type I bovine collagen matrix have been used in few case reports. ${ }^{4,8,9}$

\section{REFERENCES:}

1. Wollina U. Pyoderma gangrenosum - a review. Orphanet Journal of Rare Diseases 2007, 2: 19.

2. Iijima S, Ogawa T, Nanno Y, Tsunoda T, Kudoh K. Pyoderma gangrenosum first presenting as a recalcitrant ulcer of the ear lobe. Eur J Dermatol 2003; 13: 606-9.

3. Brunsting LA, Goeckerman WH, O' Leary PA. Pyoderma (echthyma) gangrenosum: clinical and experimental observation in five cases occurring in adults. Arch Dermatol 1930; 22: 655-80.

4. Ehling A, Karrer S, Klebl F, Andreas Schäffler, Müller-Ladner U. Therapeutic Management of Pyoderma Gangrenosum. Arthritis and rheumatism 2004; 50: 3076-3084.

5. Park HJ, Kim YC, Cinn YW,et al.: Granulomatous pyoderma gangrenosum: two unsual cases showing necrotizing granulomatous inflammation. Clin Exp Dermatol 2000, 25: 617-620.

6. Brooklyn T, Dunnill G, Probert C. Diagnosis and treatment of pyoderma gangrenosum. BMJ 2006; 333: 181-4.

7. Bisarya K, Azzopardi S, Lye G, Drew PJ. Necrotizing Fasciitis versus Pyoderma Gangrenosum: Securing the Correct Diagnosis! A Case Report and Literature Review. Jr Plastic surgery 2011; $11 ; 218-227$.

8. Wollina U, Karamfilov T. Treatment of recalcitrant ulcers in pyoderma gangrenosum with mycophenolate mofetil and autologous keratinocyte transplantation on a hyaluronic acid matrix. J Eur Acad Dermatol Venereol 2000; 14: 187-90.

9. Braun-Falco M, Stock K, Ring J, Hein R. Topical platelet-derived growth factor accelerates healing of myelodysplastic syndrome-associated pyoderma gangrenosum. Br J Dermatol 2002; 147: 829-31.

\section{AUTHORS:}

1. Sindhuri K.

2. Kallappa Herekal

3. Kariji Siddalingappa

4. Jasveen Kaur

\section{PARTICULARS OF CONTRIBUTORS:}

1. $3^{\text {rd }}$ Year Post Graduate, Department of Dermatology, venereology and Leprology, Navodaya Medical College, Raichur.

2. HOD \& Professor, Department of Dermatology, venereology and Leprology, Navodaya Medical College, Raichur.

3. Professor, Department of Dermatology, Navodaya Medical College, Raichur.
4. Assistant Professor, Department of Dermatology, Navodaya Medical College, Raichur.

NAME ADDRESS EMAIL ID OF THE CORRESPONDING AUTHOR:

Dr. Sindhuri, W/o Dr. Chetan, \# 1-11-72/24, Kakatiya Colony, Raichur, Karnataka.

Email: sindhu.kondreddy@gmail.com

Date of Submission: 12/11/2014. Date of Peer Review: 13/11/2014. Date of Acceptance: 24/11/2014. Date of Publishing: 27/11/2014. 